\title{
Effect of fermionic operators on the gauge legacy of the LHC Run I
}

\author{
Alexandre Alves, ${ }^{1}$ N. Rosa Agostinho, ${ }^{2}$ Oscar J. P. Éboli, ${ }^{3}$ and M. C. Gonzalez-Garcia ${ }^{4,2,5}$ \\ ${ }^{1}$ Departamento de Física, Universidade Federal de São Paulo, \\ UNIFESP, Diadema, 09972-270 São Paulo, Brazil \\ ${ }^{2}$ Departament de Fisica Quantica i Astrofisica and Institut de Ciencies del Cosmos, \\ Universitat de Barcelona, Diagonal 647, E-08028 Barcelona, Spain \\ ${ }^{3}$ Instituto de Física, Universidade de São Paulo, 05508-090 São Paulo, Brazil \\ ${ }^{4}$ C.N. Yang Institute for Theoretical Physics, Stony Brook University, \\ Stony Brook, New York 11794-3849, USA \\ ${ }^{5}$ Institució Catalana de Recerca i Estudis Avancats (ICREA) Pg. Lluis Companys 23, \\ 08010 Barcelona, Spain
}

(Received 11 June 2018; published 19 July 2018)

\begin{abstract}
We revisit the extraction of the triple electroweak gauge boson couplings from the Large Hadron Collider Run I data on the $W^{+} W^{-}$and $W^{ \pm} Z$ productions when the analysis also contains additional operators that modify the couplings of the gauge bosons to light quarks and the gauge boson self-energies. We work in the framework of effective Lagrangians where we consider dimension-six operators and perform a global fit to consistently take into account the bounds on these additional operators originating from the electroweak precision data. We show that the constraints on the Wilson coefficients $f_{B} / \Lambda^{2}$ and $f_{W} / \Lambda^{2}$ are modified when we include the additional operators while the limits on $f_{W W W} / \Lambda^{2}$ remain unchanged.
\end{abstract}

DOI: 10.1103/PhysRevD.98.013006

\section{INTRODUCTION}

The CERN Large Hadron Collider (LHC) has already accumulated an impressive amount of data that allow for precise tests of the Standard Model (SM), as well as, a plethora of searches for physics beyond the standard model. Moreover, the discovery of a new state [1,2], probably the Higgs boson predicted by the SM, was the first step towards the direct exploration of the electroweak symmetry breaking sector.

Within the framework of the SM, the trilinear and quartic vector-boson couplings are completely determined by the $S U(2)_{L} \otimes U(1)_{Y}$ gauge symmetry. Therefore, the scrutiny of these interactions can either lead to an additional confirmation of the SM or give some hint of the existence of new phenomena at a higher scale. The triple gauge couplings (TGC) were for the first time directly probed at LEP2 [3]. At the LHC the largest available center-of-mass energy allows for further tests of TGC. In fact, the ATLAS and CMS collaborations' studies of the $W^{+} W^{-}[4,5]$ and $W^{ \pm} Z[6,7]$ productions were already used to constrain the TGC.

Published by the American Physical Society under the terms of the Creative Commons Attribution 4.0 International license. Further distribution of this work must maintain attribution to the author(s) and the published article's title, journal citation, and DOI. Funded by SCOAP ${ }^{3}$.
The combined analysis of the LHC Run I production of electroweak gauge boson pairs performed in Ref. [8] showed that the TGC measurement is already dominated by the LHC data with better precision than the previous results from LEP2 [3]. Furthermore, the analyses of the Higgs boson properties in the framework of dimension-six effective operators can indirectly shed light on TGC $[9,10]$ by improving the accuracy of the TGC determination [11-16].

Recently, the authors of Refs. [17,18] discussed that changes in the couplings of gauge bosons to fermions, even within the constraints of electroweak precision data (EWPD), could lead to modifications of the kinematical distributions in gauge boson pair production of comparable size to the ones stemming from the purely anomalous TGC. This motivates us to revisit the analyses of the LHC Run I data on the leptonic $W^{+} W^{-}$and $W^{ \pm} Z$ productions to quantify the impact of anomalous couplings of gauge bosons to fermion pairs on the TGC bounds when consistently including in the statistical analysis the EWPD that comprise $Z$ peak observables [19], $W$ observables [20] and the Higgs mass [21].

We work in the framework of effective Lagrangians parametrizing the departures from the SM by dimension-six operators. Altogether, as described in Sec. II, the combined analysis of Run I and EWPD data comprises a total of 11 operators of which a subset of nine enter the gauge boson pair production at the LHC via modifications of the TGC, of the gauge boson couplings to fermions, as well as, 
contributions to the oblique parameters. Section III contains the details of our analyses while our results are presented in Sec. IV. They show that the largest impact on the LHC Run I constraints on TGC is on the operator $\mathcal{O}_{B}$ for which its 95\% C.L. allowed range shifts and it also becomes $\sim 30 \%$ wider. The impact on $\mathcal{O}_{W}$ is somewhat smaller while the constraints on $\mathcal{O}_{W W W}$ are not affected by the inclusion of the additional operators. We summarize and discuss our results in Sec. V.

\section{DIMENSION-SIX OPERATORS}

In this work we are interested in deviations from the Standard Model relevant to gauge boson pair production at the LHC. We parametrize those in terms of higherdimension operators as

$$
\mathcal{L}_{\text {eff }}=\mathcal{L}_{\mathrm{SM}}+\sum_{n>4, j} \frac{f_{n, j}}{\Lambda^{n-4}} \mathcal{O}_{n, j},
$$

where the $\mathcal{O}_{n, j}$ operators are linearly invariant under the SM gauge group $S U(3)_{C} \otimes S U(2)_{L} \otimes U(1)_{Y}$. Here we assume $C$ and $P$ conservation. The first operators that impact the LHC physics are of $n=6$, i.e., dimension six. The most general dimension-six operator basis respecting the SM gauge symmetry, as well as baryon and lepton number conservation, contains 59 independent operators, up to flavor and Hermitian conjugation [22,23]. Since the $S$-matrix elements are unchanged by the use of the equations of motion (EOM), there is a freedom in the choice of basis [2427]. Here we work in that of Hagiwara, Ishihara, Szalapski, and Zeppenfeld [28,29] for the pure bosonic operators.

\section{A. Bosonic operators}

There are nine $C$ - and $P$-conserving dimension-six operators in our basis involving only bosons that take part at tree level in two-to-two scattering of gauge and Higgs bosons after we employ the EOM to eliminate redundant operators [30]. Of those, five contribute to electroweak

$$
\begin{aligned}
\mathcal{O}_{\Phi L, i j}^{(1)} & =\Phi^{\dagger}\left(i \stackrel{\leftrightarrow}{D}_{\mu} \Phi\right)\left(\bar{L}_{i} \gamma^{\mu} L_{j}\right), \\
\mathcal{O}_{\Phi Q, i j}^{(1)} & =\Phi^{\dagger}\left(i \stackrel{\leftrightarrow}{D}_{\mu} \Phi\right)\left(\bar{Q}_{i} \gamma^{\mu} Q_{j}\right), \\
\mathcal{O}_{\Phi u, i j}^{(1)} & =\Phi^{\dagger}\left(i \stackrel{\leftrightarrow}{D}_{\mu} \Phi\right)\left(\bar{u}_{R_{i}} \gamma^{\mu} u_{R_{j}}\right), \\
\mathcal{O}_{\Phi u d, i j}^{(1)} & =\tilde{\Phi}^{\dagger}\left(i \stackrel{\leftrightarrow}{D}_{\mu} \Phi\right)\left(\bar{u}_{R_{i}} \gamma^{\mu} d_{R_{j}}+\text { H.c. }\right),
\end{aligned}
$$

where we defined $\tilde{\Phi}=i \sigma_{2} \Phi^{*}, \Phi^{\dagger} \stackrel{\leftrightarrow}{D}_{\mu} \Phi=\Phi^{\dagger} D_{\mu} \Phi-\left(D_{\mu} \Phi\right)^{\dagger} \Phi$ and $\Phi^{\dagger} \stackrel{\leftrightarrow}{D}_{\mu}^{a} \Phi=\Phi^{\dagger} T^{a} D_{\mu} \Phi-\left(D_{\mu} \Phi\right)^{\dagger} T^{a} \Phi$ with $T^{a}=\sigma^{a} / 2$. We have also used the notation of $L$ for the lepton doublet, $Q$ for the quark doublet and $f_{R}$ for the $S U(2)_{L}$ singlet fermions, where $i, j$ are family indices.

In order to avoid the existence of blind directions [31,32] in the analyses of the EWPD we use the freedom associated gauge boson pair production at LHC after finite renormalization effects are accounted for. In particular there is just one operator that contains exclusively gauge bosons

$$
\mathcal{O}_{W W W}=\operatorname{Tr}\left[\hat{W}_{\mu}^{\nu} \hat{W}_{\nu}^{\rho} \hat{W}_{\rho}^{\mu}\right] .
$$

In addition there are four dimension-six operators that include Higgs and electroweak gauge fields

$$
\begin{aligned}
\mathcal{O}_{W} & =\left(D_{\mu} \Phi\right)^{\dagger} \hat{W}^{\mu \nu}\left(D_{\nu} \Phi\right), & \mathcal{O}_{B} & =\left(D_{\mu} \Phi\right)^{\dagger} \hat{B}^{\mu \nu}\left(D_{\nu} \Phi\right), \\
\mathcal{O}_{B W} & =\Phi^{\dagger} \hat{B}_{\mu \nu} \hat{W}^{\mu \nu} \Phi, & \mathcal{O}_{\Phi, 1} & =\left(D_{\mu} \Phi\right)^{\dagger} \Phi \Phi^{\dagger}\left(D^{\mu} \Phi\right) .
\end{aligned}
$$

Here $\Phi$ stands for the Higgs doublet and $\sigma^{a}$ are the Pauli matrices. We have also adopted the notation $\hat{B}_{\mu \nu} \equiv i\left(g^{\prime} / 2\right) B_{\mu \nu}$ and $\hat{W}_{\mu \nu} \equiv i(g / 2) \sigma^{a} W_{\mu \nu}^{a}$, with $g$ and $g^{\prime}$ being the $S U(2)_{L}$ and $U(1)_{Y}$ gauge couplings respectively.

The first three operators in Eqs. (2.2) and (2.3) directly modify the TGC, thus, affecting the $f \bar{f} \rightarrow V V$ scattering, where $V$ stands for the electroweak gauge bosons. On the other hand, the operator $\mathcal{O}_{B W}\left(\mathcal{O}_{\Phi, 1}\right)$ is associated with the $S(T)$ oblique parameter and is constrained by the EWPD. However, these operators also modify the TGC once the Lagrangian is canonically normalized.

\section{B. Operators with fermions}

Our operator basis contains 40 independent fermionic operators, barring flavor indices, that conserve $C, P$ and baryon number and that do not involve gluon fields. Of these operators there are 28 of them that do not take part in our analyses since they either modify Higgs couplings to fermions or are contact interactions. Moreover, we also do not consider six that give rise to dipole interactions for the gauge bosons. Therefore, the operators that contribute to the processes analyzed here are

$$
\begin{aligned}
\mathcal{O}_{\Phi L, i j}^{(3)} & =\Phi^{\dagger}\left(i \stackrel{\leftrightarrow}{a}{ }_{\mu}^{a} \Phi\right)\left(\bar{L}_{i} \gamma^{\mu} T_{a} L_{j}\right), \\
\mathcal{O}_{\Phi Q, i j}^{(3)} & =\Phi^{\dagger}\left(i \stackrel{\leftrightarrow}{D_{\mu}} \Phi\right)\left(\bar{Q}_{i} \gamma^{\mu} T_{a} Q_{j}\right), \\
\mathcal{O}_{\Phi d, i j}^{(1)} & =\Phi^{\dagger}\left(i \stackrel{\leftrightarrow}{D_{\mu}} \Phi\right)\left(\bar{d}_{R_{i}} \gamma^{\mu} d_{R_{j}}\right), \\
\mathcal{O}_{\Phi e, i j}^{(1)} & =\Phi^{\dagger}\left(i \stackrel{\leftrightarrow}{D_{\mu}} \Phi\right)\left(\bar{e}_{R_{i}} \gamma^{\mu} e_{R_{j}}\right),
\end{aligned}
$$

with the use of EOM to remove from our basis the following combination of operators [30]:

$$
\sum_{i} \mathcal{O}_{\Phi L, i i}^{(1)}, \quad \text { and } \quad \sum_{i} \mathcal{O}_{\Phi L, i i}^{(3)} .
$$

Furthermore, to prevent the generation of too large flavor violation, in what follows we assume no generation mixing 
in the above operators. For the same reason we will work under the assumption that the coefficient of the potential source of additional flavor violation, $\mathcal{O}_{\Phi u d, i j}^{(1)}$, is suppressed and can be neglected. ${ }^{1}$ Also for simplicity we consider the operators to be generation independent. In this case the operators $\mathcal{O}_{\Phi L}^{(1)}$ and $\mathcal{O}_{\Phi L}^{(3)}$ are removed by the use of the EOM. Therefore, in our basis, only the operator $\mathcal{O}_{\Phi e, i j}^{(1)}$ modifies the $Z$ coupling to leptons, while there are additional contributions to the $Z$-quark pair vertices originating from $\mathcal{O}_{\Phi u, i j}^{(1)}, \mathcal{O}_{\Phi d, i j}^{(1)}, \mathcal{O}_{\Phi Q, i j}^{(1)}$, and $\mathcal{O}_{\Phi Q, i j}^{(3)}$. Moreover, the $W$ coupling to fermions receives extra contributions from $\mathcal{O}_{\Phi Q, i j}^{(3)}$ and $\mathcal{O}_{\Phi u d, i j}^{(1)}$.

The operators $\mathcal{O}_{B W}, \mathcal{O}_{\Phi, 1}, \mathcal{O}_{\Phi Q}^{(1)}, \mathcal{O}_{\Phi Q}^{(3)}, \mathcal{O}_{\Phi Q}^{(1)}, \mathcal{O}_{\Phi u}^{(1)}$, $\mathcal{O}_{\Phi d}^{(1)}$, and $\mathcal{O}_{\Phi e}^{(1)}$ can be bounded by the EWPD, in particular from $Z$-pole and $W$-pole observables [33]. In this work we focus on fermionic operators most relevant for gauge boson pair production at the LHC which are those leading to modifications of the quark couplings to gauge bosons and we will not consider $\mathcal{O}_{\Phi e}^{(1)}$ in our TGC analysis but it is kept in the EWPD studies. Furthermore, for completeness, we also include the effect of the dimension-six four-fermion operator contributing with a finite renormalization to the Fermi constant

$$
\mathcal{O}_{L L L L}=\left(\bar{L} \gamma^{\mu} L\right)\left(\bar{L} \gamma^{\mu} L\right)
$$

Altogether the effective Lagrangian considered in this work reads

$$
\begin{aligned}
\mathcal{L}_{\text {eff }}= & \mathcal{L}_{\mathrm{SM}}+\frac{f_{W W W}}{\Lambda^{2}} \mathcal{O}_{W W W}+\frac{f_{W}}{\Lambda^{2}} \mathcal{O}_{W}+\frac{f_{B}}{\Lambda^{2}} \mathcal{O}_{B} \\
& +\frac{f_{B W}}{\Lambda^{2}} \mathcal{O}_{B W}+\frac{f_{\Phi, 1}}{\Lambda^{2}} \mathcal{O}_{\Phi, 1}+\frac{f_{\Phi Q}^{(1)}}{\Lambda^{2}} \mathcal{O}_{\Phi Q}^{(1)}+\frac{f_{\Phi Q}^{(3)}}{\Lambda^{2}} \mathcal{O}_{\Phi Q}^{(3)} \\
& +\frac{f_{\Phi u}^{(1)}}{\Lambda^{2}} \mathcal{O}_{\Phi u}^{(1)}+\frac{f_{\Phi d}^{(1)}}{\Lambda^{2}} \mathcal{O}_{\Phi d}^{(1)}+\frac{f_{\Phi e}^{(1)}}{\Lambda^{2}} \mathcal{O}_{\Phi e}^{(1)}+\frac{f_{L L L L}}{\Lambda^{2}} \mathcal{O}_{L L L L} .
\end{aligned}
$$

\section{Lorentz- and $U(1)_{e m}$-invariant parametrization}

After accounting for finite renormalization effects, the part of the Lagrangian (2.7) relevant for our analyses can be cast in a Lorentz- and $U(1)_{e m}$-invariant form as

\footnotetext{
${ }^{1}$ This operator contributes only to the right-handed coupling of the $W$, and therefore it does not interfere with the SM amplitudes and, at linear order, is not constrained by the EWPD.
}

$$
\begin{aligned}
\Delta \mathcal{L}_{f, V} \equiv & -i e \Delta \kappa_{\gamma} W_{\mu}^{+} W_{\nu}^{-} \gamma^{\mu \nu}-\frac{i e \lambda_{\gamma}}{2 M_{W}^{2}} W_{\mu \nu}^{+} W^{-\nu \rho} \gamma_{\rho}^{\mu} \\
& -\frac{i e \mathrm{c}_{\mathrm{W}} \lambda_{\mathrm{Z}}}{2 M_{W}^{2}} W_{\mu \nu}^{+} W^{-\nu \rho} Z_{\rho}^{\mu}-i e \mathrm{c}_{\mathrm{W}} \Delta \kappa_{\mathrm{Z}} \mathrm{W}_{\mu}^{+} \mathrm{W}_{\nu}^{-} \mathrm{Z}^{\mu \nu} \\
& -i e \mathrm{c}_{\mathrm{W}} \Delta \mathrm{g}_{1}^{\mathrm{Z}}\left(\mathrm{W}_{\mu \nu}^{+} \mathrm{W}^{-\mu} \mathrm{Z}^{\nu}-\mathrm{W}_{\mu}^{+} \mathrm{Zn}_{\nu} \mathrm{W}^{-\mu \nu}\right) \\
& -\frac{e}{\mathrm{~S}_{\mathrm{W}} \mathrm{c}_{\mathrm{W}}} Z_{\mu} \sum_{f} \bar{\psi}^{f} \gamma^{\mu}\left[\Delta g_{L}^{f} P_{L}+\Delta g_{R}^{f} P_{R}\right] \psi^{f} \\
& -\frac{e}{\sqrt{2} \mathrm{~s}_{\mathrm{W}}}\left[W _ { \mu } ^ { + } \left(\bar{\psi}^{u} \gamma^{\mu} \Delta g_{W L}^{u d} P_{L} \psi^{d}\right.\right. \\
& \left.\left.+\bar{\psi}^{\nu} \gamma^{\mu} \Delta g_{W L}^{e \nu} P_{L} \psi^{e}\right)+ \text { H.c. }\right],
\end{aligned}
$$

where $P_{L, R}$ are the chirality projectors. The TGC effective couplings are

$$
\begin{aligned}
\Delta \kappa_{\gamma} & =\frac{e^{2} v^{2}}{8 \mathrm{~s}_{\mathrm{W}}^{2} \Lambda^{2}}\left(f_{W}+f_{B}-2 f_{B W}\right), \\
\Delta g_{1}^{Z} & =\frac{e^{2} v^{2}}{8 \mathrm{~s}_{\mathrm{W}}^{2} \mathrm{c}_{\mathrm{W}}^{2} \Lambda^{2}}\left(f_{W}+\frac{2 \mathrm{~s}_{\mathrm{W}}^{2}}{\mathrm{c}_{2 \theta_{\mathrm{W}}}} f_{B W}\right)-\frac{1}{4 \mathrm{c}_{2 \theta_{\mathrm{W}}}} \frac{v^{2}}{\Lambda^{2}} f_{\Phi, 1}, \\
\Delta \kappa_{Z} & =\Delta g_{1}^{Z}-\frac{\mathrm{s}_{\mathrm{W}}^{2}}{\mathrm{c}_{\mathrm{W}}^{2}} \Delta \kappa_{\gamma}, \\
\lambda_{\gamma} & =\lambda_{Z}=\frac{3 e^{2} M_{W}^{2}}{2 \mathrm{~s}_{\mathrm{W}}^{2} \Lambda^{2}} f_{W W W},
\end{aligned}
$$

where $c_{W}\left(s_{W}\right)$ stands for the cosine (sine) of the weak mixing angle and $c_{2 \theta_{\mathrm{W}}}$ is the cosine of twice this angle. Notice that there are only three independent TGC due to the linear realization of the $S U(2)_{L} \otimes U(1)_{Y}$ symmetry in the dimension-six operators. The effective couplings of the fermions can be written as

$$
\begin{aligned}
& \Delta g_{L, R}^{f}=g_{L, R}^{f} \Delta g_{1}+Q^{f} \Delta g_{2}+\Delta \tilde{g}_{L, R}^{f}, \\
& \Delta g_{W L}^{f f^{\prime}}=\Delta g_{W}+\Delta \tilde{g}_{W L}^{f f^{\prime}},
\end{aligned}
$$

where $g_{L}^{f}=T_{3}^{f}-\mathrm{s}_{\mathrm{W}}^{2} Q^{f}$ and $g_{R}^{f}=-\mathrm{s}_{\mathrm{W}}^{2} Q^{f}$ are the $\mathrm{SM}$ couplings. The first contributions to these anomalous couplings originate from finite renormalizations due to $\mathcal{O}_{B W}$ and $\mathcal{O}_{\Phi, 1}$

$$
\begin{aligned}
\Delta g_{1} & =\frac{1}{2}(\alpha T), \quad \Delta g_{2}=\frac{\mathrm{s}_{\mathrm{W}} 2}{\mathrm{c}_{2 \theta_{\mathrm{W}}}}\left(\mathrm{c}_{\mathrm{W}}^{2}(\alpha T)-\frac{1}{4 \mathrm{~s}_{\mathrm{W}^{2}}} \alpha S\right), \\
\Delta g_{W} & =\frac{\mathrm{c}_{\mathrm{W}}^{2}}{2 \mathrm{c}_{2 \theta_{\mathrm{W}}}} \alpha T-\frac{1}{4 \mathrm{c}_{2 \theta_{\mathrm{W}}}} \alpha S
\end{aligned}
$$

with the oblique parameters given by

$$
\alpha S=-e^{2} \frac{v^{2}}{\Lambda^{2}} f_{B W}, \quad \alpha T=-\frac{1}{2} \frac{v^{2}}{\Lambda^{2}} f_{\Phi, 1} .
$$

The fermionic dimension-six operators in Eq. (2.7) give rise to additional contributions 


$$
\begin{array}{rlrl}
\Delta \tilde{g}_{L}^{u} & =-\frac{v^{2}}{8 \Lambda^{2}}\left(4 f_{\Phi Q}^{(1)}-f_{\Phi Q}^{(3)}\right), & \Delta \tilde{g}_{R}^{u}=-\frac{v^{2}}{2 \Lambda^{2}} f_{\Phi u}^{(1)} \\
\Delta \tilde{g}_{L}^{d}=-\frac{v^{2}}{8 \Lambda^{2}}\left(4 f_{\Phi Q}^{(1)}+f_{\Phi Q}^{(3)}\right), & \Delta \tilde{g}_{R}^{d}=-\frac{v^{2}}{2 \Lambda^{2}} f_{\Phi d}^{(1)} \\
\Delta \tilde{g}_{W L}^{u d}=\frac{v^{2}}{4 \Lambda^{2}} f_{\Phi Q}^{(3)}, & \Delta \tilde{g}_{R}^{e}=-\frac{v^{2}}{2 \Lambda^{2}} f_{\Phi e}^{(1)}
\end{array}
$$

The anomalous TGC and gauge boson interactions with quarks modify the high-energy behavior of the scattering of quark pairs into two electroweak gauge bosons since the anomalous interactions can spoil the cancellations built into the SM. For the $W^{+} W^{-}$and $W^{ \pm} Z$ channels the leading scattering amplitudes in the helicity basis are

$$
\begin{aligned}
& A\left(d_{-} \bar{d}_{+} \rightarrow W_{0}^{+} W_{0}^{-}\right) \\
& \quad=i \frac{s}{\Lambda^{2}} \sin \theta\left\{-\frac{g^{2}}{24 c_{W}^{2}}\left(3 c_{W}^{2} f_{W}-s_{W}^{2} f_{B}\right)+\frac{1}{4}\left(f_{\Phi Q}^{(3)}-4 f_{\Phi Q}^{(1)}\right)\right\},
\end{aligned}
$$

$$
A\left(d_{-} \bar{d}_{+} \rightarrow W_{ \pm}^{+} W_{ \pm}^{-}\right)=-i \frac{s}{\Lambda^{2}} \sin \theta \frac{3 g^{4}}{8} f_{W W W}
$$

$$
A\left(d_{+} \bar{d}_{-} \rightarrow W_{0}^{+} W_{0}^{-}\right)=-i \frac{s}{\Lambda^{2}} \sin \theta\left\{\frac{g^{2} s_{W}^{2}}{12 c_{W}^{2}} f_{B}+f_{\Phi d}^{(1)}\right\},
$$

$$
\begin{aligned}
& A\left(u_{-} \bar{u}_{+} \rightarrow W_{0}^{+} W_{0}^{-}\right) \\
& \quad=i \frac{s}{\Lambda^{2}} \sin \theta\left\{\frac{g^{2}}{24 c_{W}^{2}}\left(3 c_{W}^{2} f_{W}+s_{W}^{2} f_{B}\right)-\frac{1}{4}\left(f_{\Phi Q}^{(3)}+4 f_{\Phi Q}^{(1)}\right)\right\},
\end{aligned}
$$

$$
\begin{aligned}
A\left(u_{+} \bar{u}_{-} \rightarrow W_{0}^{+} W_{0}^{-}\right)=i \frac{s}{\Lambda^{2}} \sin \theta\left\{\frac{g^{2} s_{W}^{2}}{6 c_{W}^{2}} f_{B}-f_{\Phi u}^{(1)}\right\} & \\
A\left(u_{-} \bar{u}_{+} \rightarrow W_{ \pm}^{+} W_{ \pm}^{-}\right) & =A\left(d_{-} \bar{u}_{+} \rightarrow Z_{ \pm} W_{ \pm}^{-}\right) \\
& =i \frac{s}{\Lambda^{2}} \sin \theta \frac{3 g^{4}}{8} f_{W W W}
\end{aligned}
$$

$A\left(d_{-} \bar{u}_{+} \rightarrow W_{0}^{-} Z_{0}\right)=i \frac{s}{\Lambda^{2}} \sin \theta\left\{\frac{g^{2}}{4 \sqrt{2}} f_{W}-\frac{1}{2 \sqrt{2}} f_{\Phi Q}^{(3)}\right\}$,

where $s$ stands for the center-of-mass energy and $\theta$ is the polar angle in the center-of-mass frame.

We notice that the leptonic operator $\mathcal{O}_{\Phi e}^{(1)}$ does not contribute to the gauge boson production amplitudes at the LHC. It only contributes to the decay rate of the $Z$ boson in the $Z W$ channels and in the narrow-width approximation its effect is subdominant. We will not consider it in the TGC analysis but it is kept in the EWPD analysis.

\section{ANALYSIS FRAMEWORK}

\begin{tabular}{|c|c|c|c|c|c|c|}
\hline Channel $(a)$ & Distribution & \# bins $\left(N_{b}\right)$ & Data set & $\sigma_{\text {sig }}$ & $\sigma_{\text {bck }}$ & $\sigma_{i, \text { unc }}$ \\
\hline$W W \rightarrow \ell^{+} \ell^{\prime-}+\mathbb{E}_{T}(0 j)$ & $p_{T}^{\text {leading,lepton }}$ & 3 & ATLAS $8 \mathrm{TeV}, 20.3 \mathrm{fb}^{-1}$ [4] & 0.049 & 0.02 & $0.08-0.14$ \\
\hline$W W \rightarrow \ell^{+} \ell^{(\prime)-}+\mathbb{E}_{T}(0 j)$ & $m_{\ell \ell^{(\prime)}}$ & 8 & CMS $8 \mathrm{TeV}, 19.4 \mathrm{fb}^{-1}[5]$ & 0.069 & 0.02 & $0.01-0.08$ \\
\hline$W Z \rightarrow \ell^{+} \ell^{-} \ell^{(\prime) \pm}$ & $m_{T}^{W Z}$ & 6 & ATLAS $8 \mathrm{TeV}, 20.3 \mathrm{fb}^{-1}[6]$ & 0.1 & 0.02 & $0.12-0.18$ \\
\hline$W Z \rightarrow \ell^{+} \ell^{-} \ell^{(\prime) \pm}+\mathbb{E}_{T}$ & $Z$ candidate $p_{T}^{\ell \ell}$ & 10 & CMS $8 \mathrm{TeV}, 19.6 \mathrm{fb}^{-1}$ [7] & 0.15 & 0.02 & $0.15-0.25$ \\
\hline
\end{tabular}

In order to constrain the parameters in the effective Lagrangian in Eq. (2.7) we study the $W^{+} W^{-}$and $W^{ \pm} Z$ productions in the leptonic channel since these are the measurements with the highest sensitivity for charged triple gauge boson vertices. In doing so we consider the same kinematic distributions employed by the experiments for their anomalous gauge boson coupling analyses which allows us to validate our results against the bounds obtained by the experiments in each of the final states. More specifically, the channels that we analyze and their kinematical distributions are
For each experiment and channel, we extract from the experimental publications the observed event rates in each bin, $N_{i, \mathrm{~d}}^{a}$, as well as the background expectations $N_{i \text {,bck }}^{a}$, and the $\mathrm{SM} W^{+} W^{-}\left(W^{ \pm} Z\right)$ predictions, $N_{i, \mathrm{sm}}^{a}$.

The procedure to obtain the relevant kinematical distributions predicted by Eq. (2.7) is as follows. First we simulate the $W^{+} W^{-}$and $W^{ \pm} Z$ productions using MADGRAPH5 [34] with the UFO files for our effective Lagrangian generated with FEYnRules [35,36]. We employ PyTHIA6.4 [37] to perform the parton shower, while the fast detector simulation is carried out with DELPHES [38]. In order to account for higher-order corrections and additional detector effects we simulate SM $W^{+} W^{-}$and $W^{ \pm} Z$ productions in the fiducial region requiring the same cuts and isolation criteria adopted by the corresponding ATLAS and CMS studies, and normalize our results bin by bin to the experimental collaboration predictions for the kinematical distributions under consideration. Then we apply these correction factors to our 

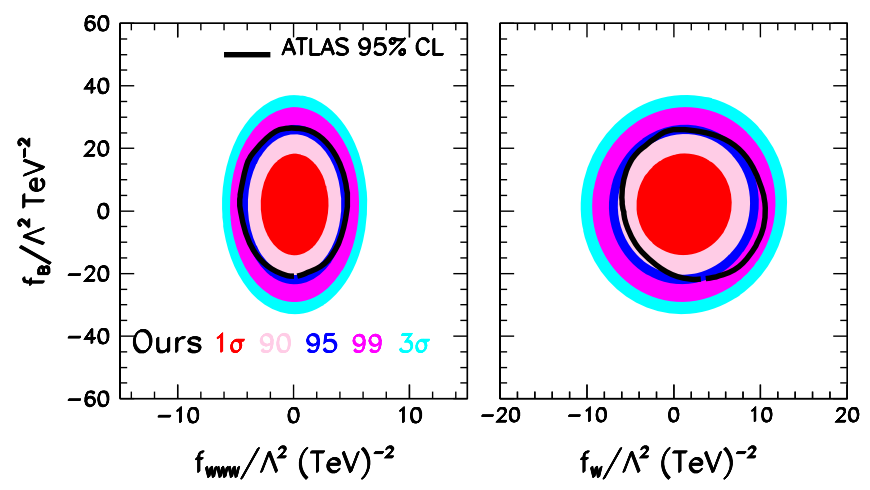

FIG. 1. Allowed regions in the planes $f_{B} / \Lambda^{2} \otimes f_{W W W} / \Lambda^{2}$ (left panel) and $f_{B} / \Lambda^{2} \otimes f_{W} / \Lambda^{2}$ (right panel) at $1 \sigma, 95 \%, 99 \%$, and $3 \sigma$ C.L. The black line stands for the border of the $95 \%$ C.L. allowed region obtained by ATLAS [4].

simulated $W V$ distributions in the presence of the anomalous couplings. This procedure yields our predicted number of signal events in each bin $i$ for the " $a$ " channel, $N_{i, \text { sig }}^{a \text {, nosys }}$.

The statistical confrontation of these predictions with the LHC Run I data is made by means of a binned loglikelihood function based on the contents of the different bins in the relevant kinematical distribution of each channel. Depending on the number of data events in the bin we use a Poissonian or a Gaussian probability distribution for its statistical error. In constructing the loglikelihood function we simulate the effect of the systematic and theoretical uncertainties by introducing two sets of pulls: two that globally affect the predictions of the event rates in all bins in fully correlated form (which parametrize, among others, the luminosity uncertainty, and theoretical errors on the total cross section for the process and its backgrounds), and $N_{b}^{a}$ independent pulls, one per bin, to account for the bin-uncorrelated errors arising from the theoretical errors affecting the distributions, experimental energy resolutions and, in general, any energy and/or momentum dependence of the uncertainties. With this, the number of predicted events in bin $i$ for channel $a$ is $N_{i}^{a}=\left[\left(1+\xi_{\text {sig }}^{a}\right)\left(1+\xi_{i, \text { unc }}^{a}\right) N_{i, \text { sig }}^{a, \text { nosys }}+\left(1+\xi_{\text {bck }}^{a}\right) N_{i, \text { bck }}^{a}\right]$. The errors of these pulls are introduced as Gaussian bias in the log-likelihood functions and are extracted from the information given by the experiments. For completeness they are reported in the table above.

In order to validate our simulation we obtain first the 95\% C.L. allowed regions for the TGC for each channel and experiment under the same assumptions the collaboration used. For example, we present in Fig. 1 our twodimensional allowed regions using the ATLAS $W^{+} W^{-}$data and assuming that the only nonvanishing Wilson coefficients are $f_{W W W}, f_{W}$ and $f_{B}$, with two of them different from zero at a time, as in the ATLAS analysis. As seen in the figure, our results for the $95 \%$ C.L. allowed region (blue region) agree well with the one obtained by ATLAS, whose border is represented by the black curve.
When including the effect of the additional operators we must also account for their contribution to the EWPD. Our construction of the $\chi^{2}$ function for the EWPD follows the analysis in Ref. [33] to which we refer the reader for details. In brief in our EWPD analysis we fit 15 observables of which 12 are $Z$ observables [19]:

$$
\begin{array}{lllll}
\Gamma_{Z}, & \sigma_{h}^{0}, \quad \mathcal{A}_{\ell}\left(\tau^{\mathrm{pol}}\right), & R_{\ell}^{0}, & \mathcal{A}_{\ell}(\mathrm{SLD}), \\
A_{\mathrm{FB}}^{0, l}, \quad R_{c}^{0}, \quad R_{b}^{0}, & \mathcal{A}_{c}, & \mathcal{A}_{b}, & A_{\mathrm{FB}}^{0, c}, & \text { and } \\
A_{\mathrm{FB}}^{0, b}(\mathrm{SLD} / \mathrm{LEP}-\mathrm{I}), & & &
\end{array}
$$

complemented by three $W$ observables

$$
M_{W}, \quad \Gamma_{W} \quad \text { and } \quad \operatorname{Br}(W \rightarrow \ell \nu)
$$

that are, respectively, its average mass taken from Ref. [39], its width from LEP2/Tevatron [20], and the leptonic $W$ branching ratio for which the average in Ref. [39] is considered. The correlations among these inputs were presented in Ref. [19] and we take them into consideration in the analyses. The SM predictions and their uncertainties due to variations of the SM parameters were extracted from Ref. [40].

Altogether we construct a combined $\chi^{2}$ function

$$
\begin{aligned}
\chi_{\mathrm{LHC}-\mathrm{RI}+\mathrm{EWPD}}^{2} \equiv & \chi_{\mathrm{LHC}-\mathrm{RI}}^{2}\left(f_{W}, f_{B}, f_{W W W}, f_{B W}, f_{\Phi, 1}, f_{\phi, Q}^{(1)},\right. \\
& \left.f_{\phi, Q}^{(3)}, f_{\phi, u}^{(1)}, f_{\phi, d}^{(1)}\right) \\
+ & \chi_{\mathrm{EWPD}}^{2}\left(f_{B W}, f_{\Phi, 1}, f_{\phi, Q}^{(1)}, f_{\phi, Q}^{(3)}, f_{\phi, u}^{(1)},\right. \\
& \left.f_{\phi, d}^{(1)}, f_{\phi, e}^{(1)}, f_{L L L L}\right),
\end{aligned}
$$

from which we derive the allowed ranges for each coefficient or pair of coefficients after marginalization over all the others.

Finally, for comparison, we also consider the constraints from the LEP2 global analysis of TGC [3]. In order to do so we follow the procedure in Ref. [8] and construct a simplified Gaussian $\chi_{\text {LEP2 }}^{2}$ using the central values, $\sigma$ and the correlation matrix for the couplings $\Delta g_{Z}^{1}, \Delta \kappa_{\gamma}$ and $\lambda$ and their correlation coefficients from the final combined LEP2 analysis in Ref. [3] (reproduced in Table I for completeness) which was performed in terms of these effective TGC

TABLE I. $\Delta g_{1}^{Z}, \Delta \kappa_{\gamma}$ and $\lambda$ central values, standard deviations and correlation coefficients from LEP2 [3].

\begin{tabular}{lrrcr}
\hline \hline & \multicolumn{4}{c}{ LEP } \\
\cline { 2 - 5 } & \multicolumn{1}{c}{ 68\% C.L. } & \multicolumn{3}{c}{ Correlations } \\
\hline$\Delta g_{1}^{Z}$ & $0.051_{-0.032}^{+0.031}$ & 1.00 & 0.23 & -0.30 \\
$\Delta \kappa_{\gamma}$ & $-0.067_{-0.057}^{+0.061}$ & 0.23 & 1.00 & -0.27 \\
$\lambda$ & $-0.067_{-0.038}^{+0.036}$ & -0.30 & 0.27 & 1.00 \\
\hline \hline
\end{tabular}



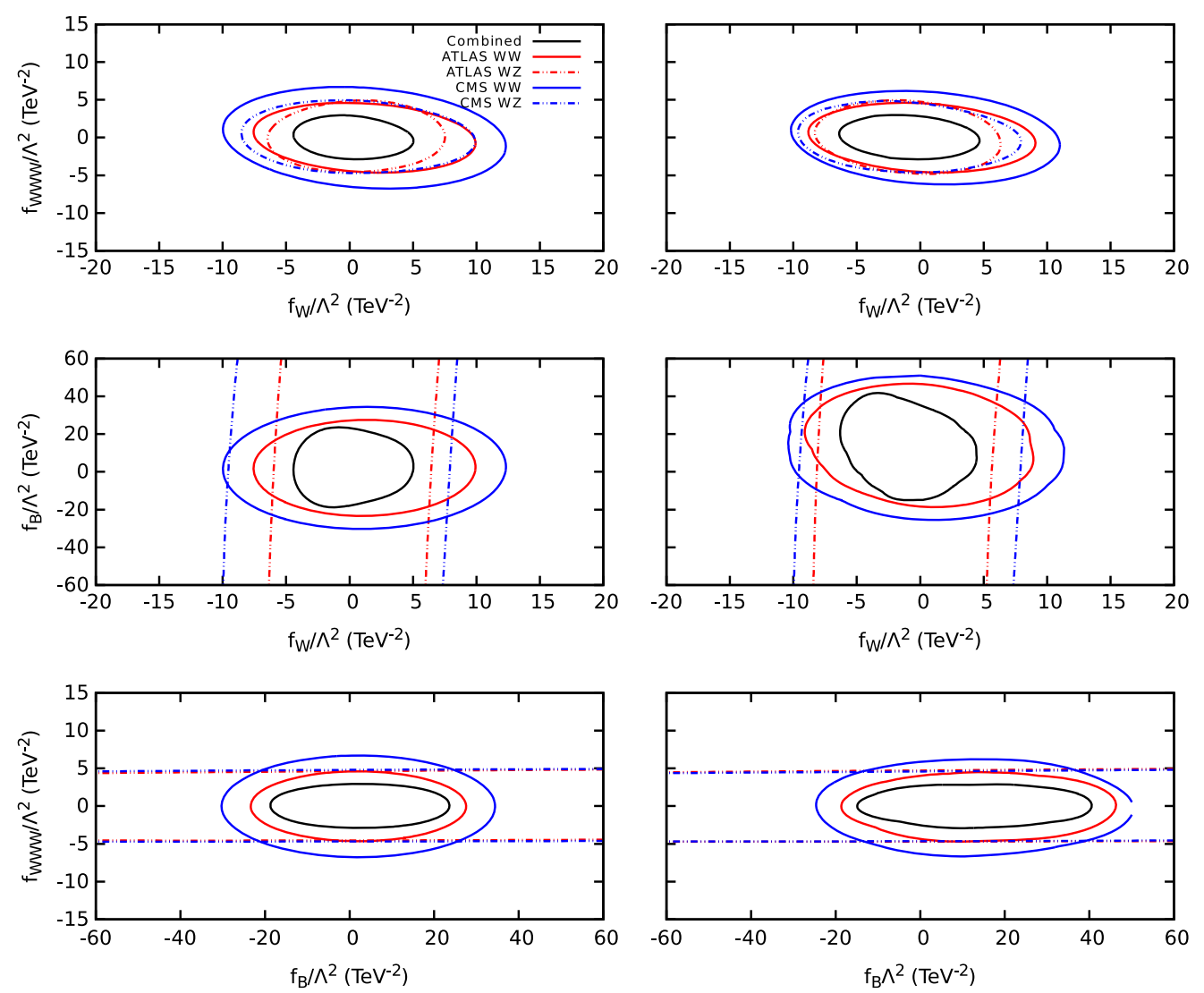

FIG. 2. Allowed $95 \%$ C.L. regions in the planes $f_{W W W} / \Lambda^{2} \otimes f_{B} / \Lambda^{2}$ (top row), $f_{B} / \Lambda^{2} \otimes f_{W} / \Lambda^{2}$ (middle row) and $f_{W W W} / \Lambda^{2} \otimes$ $f_{W} / \Lambda^{2}$ (lower row) for the different channels as labeled in the figure. In the left panels only $f_{W W W}, f_{W}$ and $f_{B}$ are considered nonzero in the fit, while the right panels display the result from the 11-parameter fit. In each case we marginalize over the undisplayed nonzero variables.

coefficients under the relations implied by the dimensionsix effective operator formalism for TGC. We notice, however, that in extracting those bounds on the effective TGC couplings, the LEP collaborations did not include the effect of fermion operators. For that reason the combination of those LEP2 bounds with our LHC Run I data and EWPD is only shown for the purpose of illustration.

\section{BOUNDS ON TRIPLE GAUGE BOSON INTERACTIONS}

We start by showing the results of our analysis in terms of the allowed ranges for the Wilson coefficients of the three "canonical" TGC operators, $f_{W W W} / \Lambda^{2}, f_{W} / \Lambda^{2}$ and $f_{B} / \Lambda^{2}$. We depict first in Fig. 2 the $95 \%$ C.L. (two d.o.f.) allowed regions in the planes $f_{B} / \Lambda^{2} \otimes f_{W} / \Lambda^{2}, f_{W W W} / \Lambda^{2} \otimes$ $f_{B} / \Lambda^{2}$ and $f_{W W W} / \Lambda^{2} \otimes f_{W} / \Lambda^{2}$ for the $W^{+} W^{-}$and $W^{ \pm} Z$ channels and for ATLAS and CMS, as well as the combination of these results. In order to assess the impact of additional operators in the TGC extraction at the LHC we first perform the "standard" analysis by fitting just these three coefficients, and setting the coefficients of all other operators to zero. The corresponding allowed regions are shown in the left panels after marginalizing over the third coefficient which is not displayed. Conversely the results of the global analysis of the LHC Run I data together with EWPD performed in terms of 11 nonzero Wilson coefficients [see Eq. (2.7)] are shown in the right panels. These regions are obtained after marginalization over the 9 undisplayed coefficients.

One salient feature of Fig. 2 is that the $W^{ \pm} Z$ bounds on the Wilson coefficient $f_{B} / \Lambda^{2}$ are much looser than the ones on $f_{W} / \Lambda^{2}$ and $f_{W W W} / \Lambda^{2}$, as expected, because $\mathcal{O}_{B}$ does not contribute to the leading term of the growth of the scattering amplitudes; see Eqs. (2.14)-(2.20).

For better comparison of the results obtained with and without including the additional operators we overlay in Fig. 3 the $1 \sigma$ and $95 \%$ C.L. allowed regions obtained combining all channels and experiments for the two scenarios. As we can see from this figure the addition of more parameters leads to the expansion of the allowed regions, as expected. Moreover, the region of $f_{B} / \Lambda^{2}$ suffers the largest shift towards positive values of this parameter while there is a small shift in the $f_{W} / \Lambda^{2}$ direction and there is no appreciable displacement along the $f_{W W W} / \Lambda^{2}$ axis. 


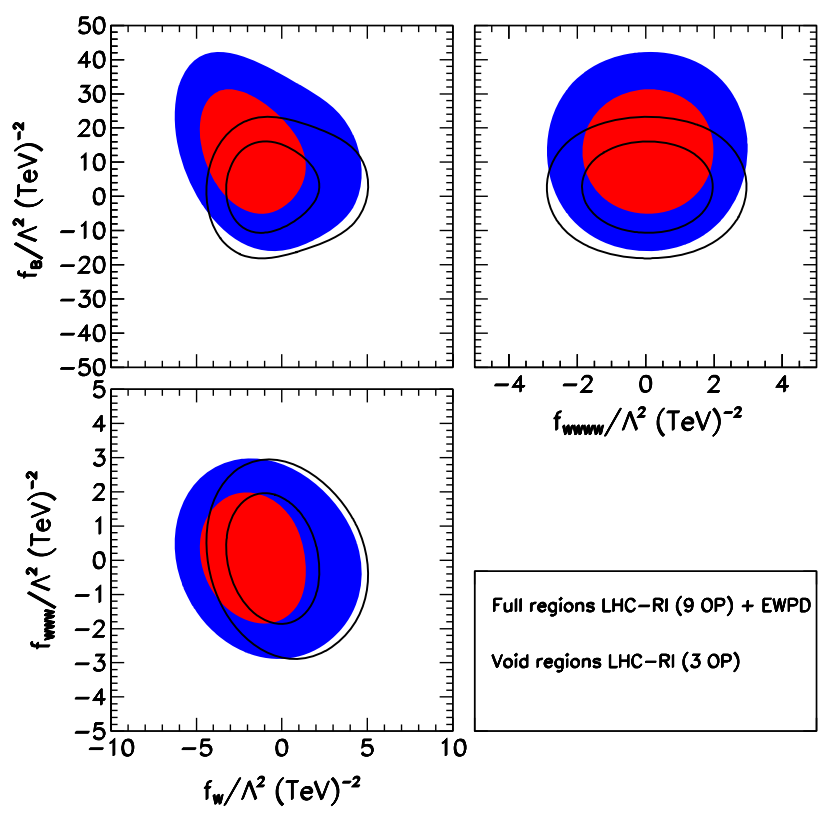

FIG. 3. $1 \sigma$ and $95 \%$ C.L. allowed regions in the planes indicated on the axes. Here we consider the $W^{+} W^{-}$and $W^{ \pm} Z$ productions and the EWPD in the analyses. The data set and parameters used are as indicated in the figure.

The corresponding dependence of the $\Delta \chi^{2}$ for the two analyses with each of the three coefficients is given in Fig. 4 and from those we read the 95\% C.L. one-dimensional allowed ranges for each coefficient given in Table II. As seen above, the $\Delta \chi^{2}$ distribution for $f_{B} / \Lambda^{2}\left(f_{W} / \Lambda^{2}\right)$ broadens and shifts to positive (negative) values when we compare the results considering only the LHC Run I data and three canonical parameters (solid black line) with the one containing additional operators also constrained by the EWPD (solid blue line). Quantitatively the effect is slightly larger for $f_{B} / \Lambda^{2}$ whose allowed range widens by about $30 \%$ versus $20 \%$ for $f_{W} / \Lambda^{2}$.
TABLE II. 95\% C.L. allowed ranges for the Wilson coefficients of the dimension-six operators that contribute to the studied processes in gauge boson pair production at the LHC. The ranges for each parameter are obtained after marginalization of the coefficients of all other operators contributing to each analysis. In particular the results given in the third and forth columns are obtained after marginalization over $f_{\phi, e}^{(1)}$ and $f_{L L L L}$ as well.

\begin{tabular}{lccc}
\hline \hline & \multicolumn{3}{c}{$95 \%$ allowed range $\left(\mathrm{TeV}^{-2}\right)$} \\
\cline { 2 - 4 } Coupling & $\begin{array}{c}\text { LHC RI } \\
(3 \text { OP })\end{array}$ & EWPD & $\begin{array}{c}\text { LHC RI } \\
(9 \text { OP })+\text { EWPD }\end{array}$ \\
\hline$f_{W}$ & $(-3.9,3.9)$ & $\ldots$ & $(-5.6,4.0)$ \\
$f_{B}$ & $(-15,20)$ & $\ldots$ & $(-11,37)$ \\
$f_{W W W}$ & $(-2.4,2.5)$ & $\ldots$ & $(-2.4,2.6)$ \\
$f_{B W}$ & $\ldots$ & $(-0.32,1.7)$ & $(-0.33,1.7)$ \\
$f_{\Phi 1}$ & $\ldots$ & $(-0.040,0.15)$ & $(-0.044,0.15)$ \\
$f_{\Phi Q}^{(1)}$ & $\ldots$ & $(-0.083,0.10)$ & $(-0.044,0.12)$ \\
$f_{\Phi Q}^{(3)}$ & $\ldots$ & $(-0.60,0.12)$ & $(-0.52,0.18)$ \\
$f_{\Phi u}^{(1)}$ & $\ldots$ & $(-0.25,0.37)$ & $(-0.19,0.42)$ \\
$f_{\Phi d}^{(1)}$ & $\ldots$ & $(-1.2,-0.13)$ & $(-0.73,0.023)$ \\
\hline \hline
\end{tabular}

The effect of each of the six additional operators on the extracted range of the three "canonical" TGC operator coefficients is illustrated in Fig. 5 where we depict the twodimensional correlations between the three TGC coefficients and the additional ones. In each panel of this figure we exhibit the $1 \sigma$ and $95 \%$ C.L. level (two d.o.f.) allowed regions after marginalizing over the remaining parameters. As we can see, $f_{B} / \Lambda^{2}$ has a significant correlation only with $f_{\Phi u}^{(1)} / \Lambda^{2}$ and to a lesser extent is (anti)correlated with $f_{\Phi Q}^{(1)} / \Lambda^{2}\left(f_{\Phi Q}^{(3)} / \Lambda^{2}\right.$ and $\left.f_{\Phi d}^{(1)} / \Lambda^{2}\right)$. This is expected as these are the operator coefficients contributing the growth of the scattering amplitudes into longitudinally polarized gauge bosons [Eqs. (2.14)-(2.20)]. In particular the correlation with $f_{\Phi u}^{(1)} / \Lambda^{2}$ can be understood from the scattering

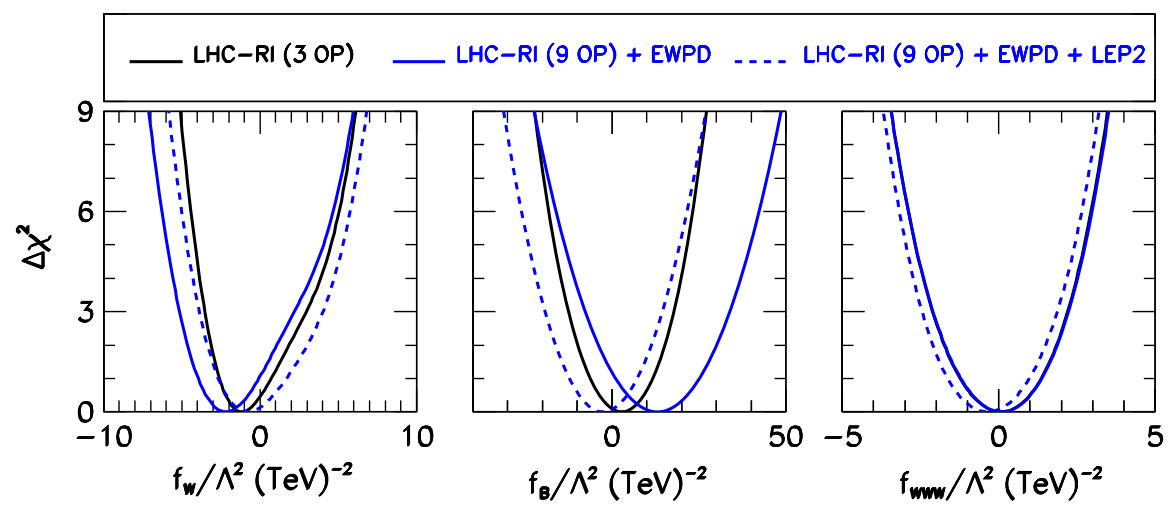

FIG. 4. $\Delta \chi^{2}$ dependence on the $f_{W} / \Lambda^{2}$ (left panel), $f_{B} / \Lambda^{2}$ (central panel) and $f_{W} / \Lambda^{2}$ (right panel) parameters after the marginalization over the remaining fit parameters. The solid black line stands for the standard TGC analysis, while the solid blue line represents the nine-parameter fit to the LHC Run I data and the EWPD. The dashed blue line differs from the solid ones just by the addition of LEP2 data on TGC. 

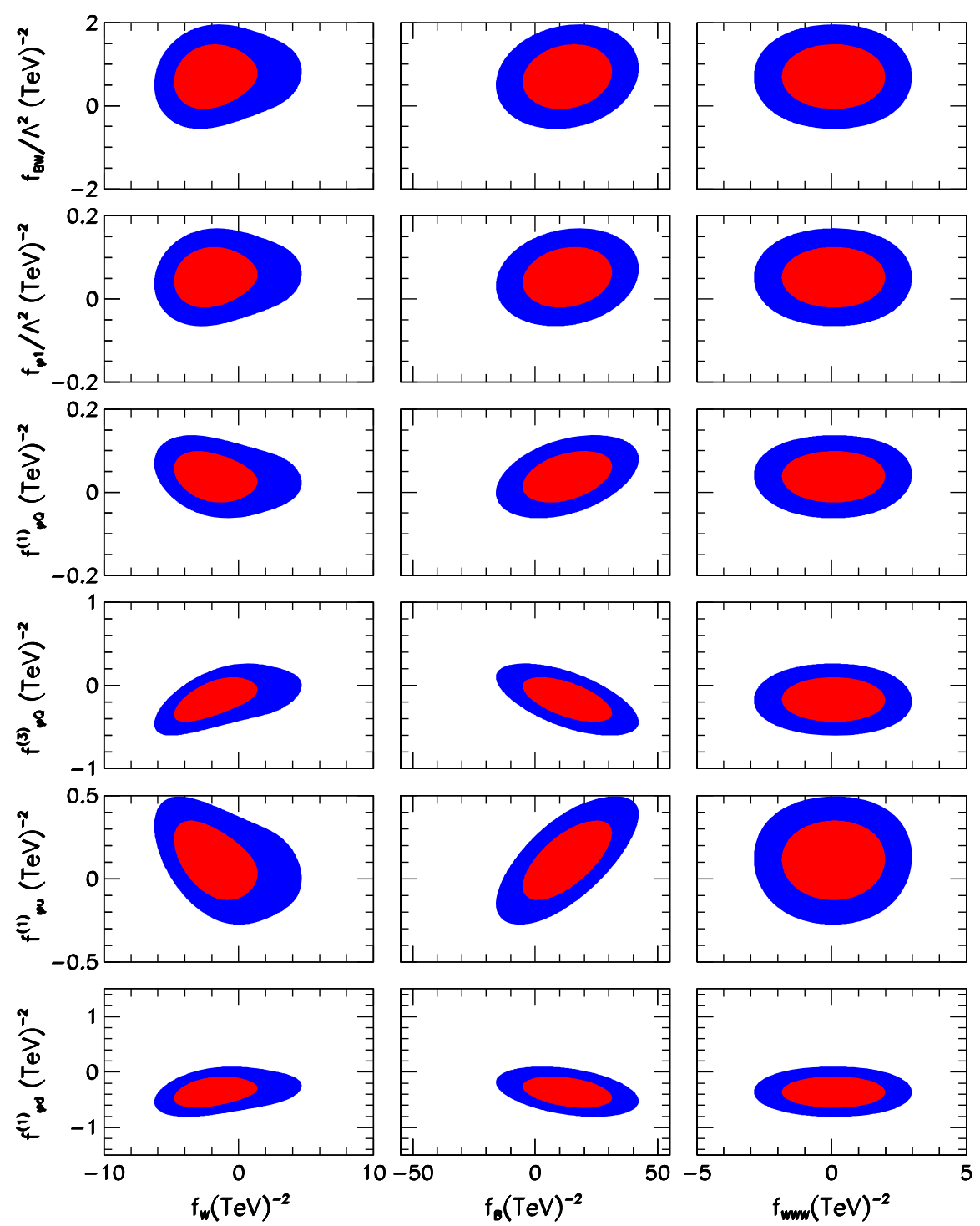

FIG. 5. $1 \sigma$ and $95 \%$ C.L. allowed regions in the planes indicated on the axes. Here we consider the $W^{+} W^{-}$and $W^{ \pm} Z$ productions and the EWPD in the analyses.

amplitude in Eq. (2.18). Similarly $f_{W} / \Lambda^{2}$ shows a stronger anticorrelation only with $f_{\Phi u}^{(1)} / \Lambda^{2}$ and to a smaller degree is correlated with $f_{\Phi Q}^{(3)} / \Lambda^{2}$ and $f_{\Phi d}^{(1)} / \Lambda^{2}$. Finally from the third column of this figure we can see that $f_{W W W} / \Lambda^{2}$ shows no correlation with the additional parameters as expected since $\mathcal{O}_{W W W}$ contributes by itself to the energy growth of the scattering amplitudes for transversely polarized gauge bosons.

The impact of the LHC diboson production data on the determination of the parameters directly constrained by the EWPD is illustrated in Fig. 6 which depicts the $\Delta \chi^{2}$ distribution as a function of these parameters where the magenta (blue) line stands for the result obtained using the EWPD (and the LHC Run I diboson production data).

The top left and middle panels of this figure show that the addition of the LHC data does not alter the constraints on the
$f_{B W} / \Lambda^{2}$ and $f_{\Phi 1} / \Lambda^{2}$ parameters. This is easy to understand since these parameters do not modify the high-energy behavior of $q \bar{q} \rightarrow V V$ amplitudes; see Eqs. (2.14)-(2.20). This is expected from $\mathcal{O}_{\Phi, 1}$ as it only contributes to the amplitudes via finite renormalization effects of the SM parameters. The operator $\mathcal{O}_{B W}$, on the other hand, also directly modifies the TGC; however, its effects on the $Z$ wave-function renormalization cancel the growth with the center-of-mass energy due to the anomalous TGC. From the top right, bottom left and middle panels we can see that the impact of the Run I data on $f_{\Phi Q}^{(1)} / \Lambda^{2}, f_{\Phi Q}^{(3)} / \Lambda^{2}$ and $f_{\Phi u}^{(1)} / \Lambda^{2}$ is marginal. $f_{\Phi d}^{(1)} / \Lambda^{2}$ is the only parameter whose $\Delta \chi^{2}$ distribution gets significantly affected. The EWPD analysis favors a nonvanishing value for $f_{\Phi d}^{(1)} / \Lambda^{2}$ at $2 \sigma$, a result driven by the $2.7 \sigma$ discrepancy between the observed $A_{\mathrm{FB}}^{0, b}$ and the SM. On the contrary no significant discrepancy is observed between 

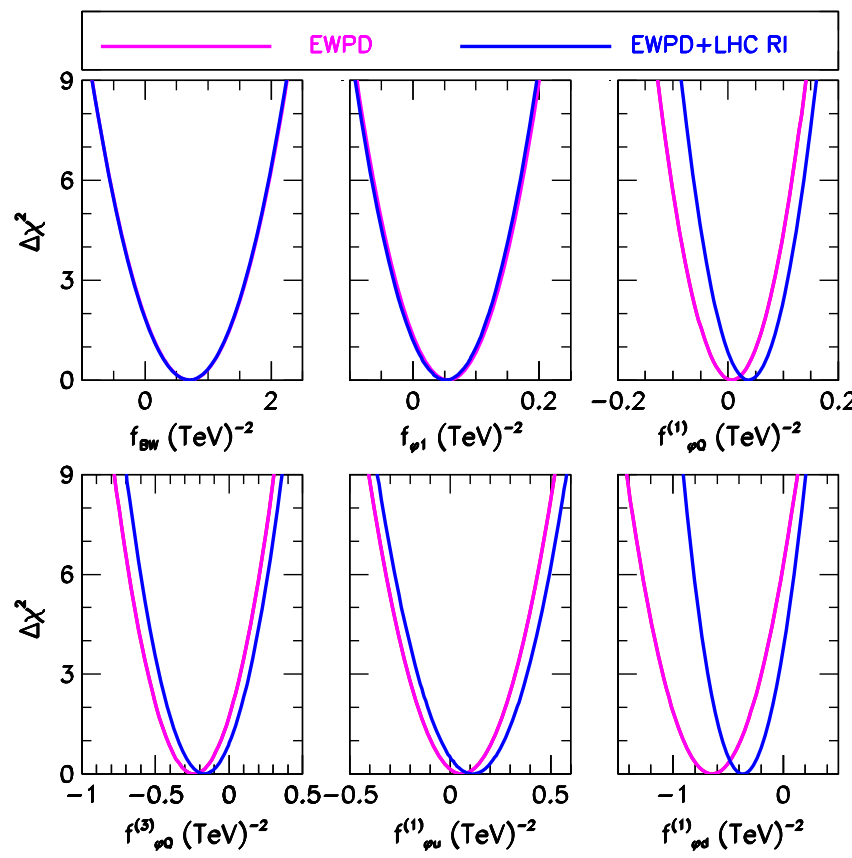

FIG. 6. $\Delta \chi^{2}$ dependence on $f_{B W} / \Lambda^{2}, f_{\Phi 1} / \Lambda^{2}, f_{\Phi Q}^{(1)} / \Lambda^{2}$, $f_{\Phi Q}^{(3)} / \Lambda^{2}, f_{\Phi u}^{(1)} / \Lambda^{2}$, and $f_{\Phi d}^{(1)} / \Lambda^{2}$ after the marginalization over the undisplayed parameters. The magenta line stands for the results using only the EWPD while the blue one is obtained considering the EWPD and LHC Run I diboson production.

the observed LHC Run I diboson data and the SM. Hence there is a shift towards zero of $f_{\Phi d}^{(1)} / \Lambda^{2}$ when including the LHC Run I data in the analysis. This slight tension results also in the reduction of the globally allowed range.

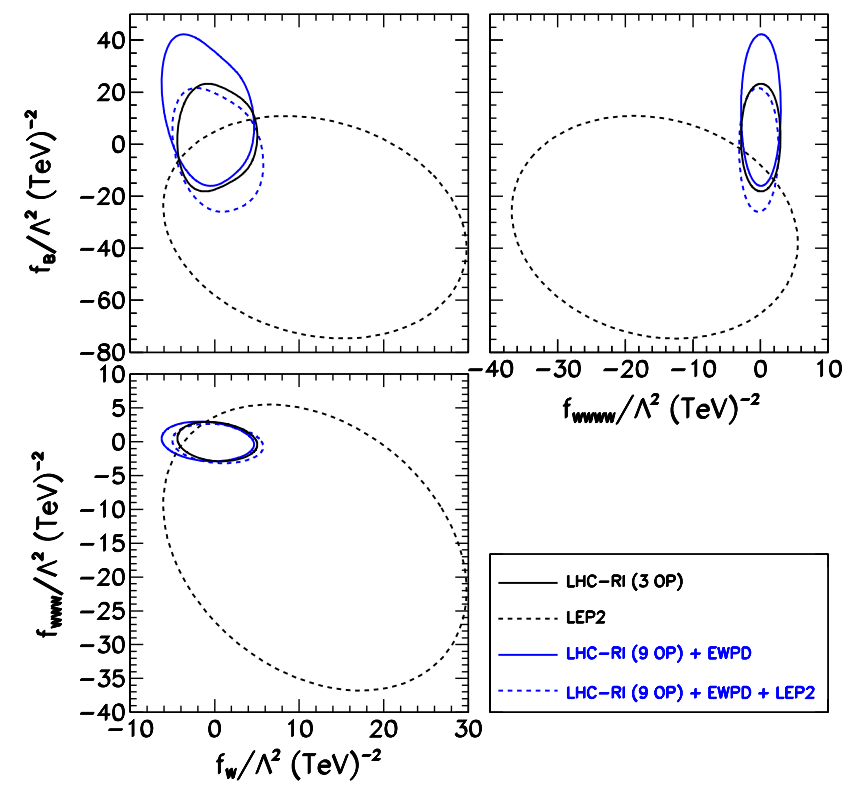

FIG. 7. Two-dimensional $95 \%$ C.L. allowed regions in the planes indicated on the axes. Here we consider the $W^{+} W^{-}$and $W^{ \pm} Z$ productions, LEP2 data on TGC and the EWPD in the analyses. The lines are as shown in the figure.
We finish this section by comparing our results with the bounds derived from LEP2 diboson data. To do so we plot in Fig. 7 the two-dimensional 95\% C.L. allowed regions for the three combinations of the canonical TGC parameters for the analysis with and without additional operators together with the LEP2 results. As shown in Ref. [8], the limits emanating from the canonical LHC Run I diboson data (black solid line) are substantially more stringent than those imposed by LEP2 (black dashed line). As seen in this figure, enlarging the number of operators in the LHC analyses, together with the EWPD, does not alter this conclusion despite the growth of the allowed regions (solid blue line). For illustration we also show in the figure the allowed regions obtained by naively combining the general LHC Run I + EWPD analysis with the LEP2 information (see discussion at the end of Sec. III). As seen, including LEP2 data in the approximation used leads to a reduction of the allowed regions in the $f_{B} / \Lambda^{2}$ direction, as well as to a shift of it towards negative values (see also the dashed blue line in Fig. 4).

\section{SUMMARY}

In this work we have quantified the impact of possible anomalous gauge couplings to quarks on the TGC determination performed using the LHC Run I diboson data. In order to carry out a statistically consistent analysis we have included in addition the EWPD to constrain the couplings between quarks and gauge bosons as well as the modifications of the gauge boson self-energies. We have worked in the framework of effective Lagrangians so our study has been performed including the 11 dimension-six operators given in Eq. (2.7).

As a summary of our findings we present in Table II the 95\% C.L. globally allowed ranges for the Wilson coefficients of the nine operators that contribute to the LHC Run I data considered. The comparison of the first and third columns of this table shows that the addition of the new operators modifies the TGC bounds on $f_{B} / \Lambda^{2}$ and $f_{W} / \Lambda^{2}$ coming from the LHC Run I diboson data. Quantitatively the effect is slightly larger for $f_{B} / \Lambda^{2}$ whose allowed range widens by about $30 \%$ versus $20 \%$ for $f_{W} / \Lambda^{2}$. The limits on $f_{W W W} / \Lambda^{2}$, on the contrary, are almost unaffected. Despite these changes, the constraints on these parameters are still dominated by the LHC Run I data, and are still substantially stronger than those obtained from LEP2 data.

We have also learned from our analyses that the LHC Run I diboson data is not precise enough to yield substantial information on the gauge couplings to quarks in addition to what is already known from EWPD; compare the second and third columns of Table II. The only apparent exception is $f_{\Phi d}^{(1)} / \Lambda^{2}$ which in the considered family universal scenario is driven to be nonzero in the EWPD analysis by the discrepancy between the measured $A_{\mathrm{FB}}^{0, b}$ at LEP/SLC and the SM while LHC Run I data shows no evidence of any deviation 
with respect to the SM. Nevertheless, these results allow us to foresee that diboson production at the LHC will play an important role in the analyses of anomalous couplings of gauge bosons to quarks as the LHC increases its integrated luminosity. Hence global analyses of LHC data and EWPD are becoming necessary for a consistent determination of the Wilson coefficients of the full set of dimension-six operators.

\section{ACKNOWLEDGMENTS}

We thank Tyler Corbett for discussions. O. J. P. E. and N. R. A. want to thank the group at SUNY at Stony Brook for the hospitality during the final stages of this work. This work is supported in part by Conselho Nacional de
Desenvolvimento Científico e Tecnológico (CNPq) and by Fundação de Amparo à Pesquisa do Estado de São Paulo (FAPESP) Grants No. 2012/10095-7 and No. 2017/061095, by USA-NSF Grant No. PHY-1620628, by EU Networks FP10 ITN ELUSIVES (H2020-MSCA-ITN-2015-674896) and INVISIBLES-PLUS (H2020-MSCA-RISE-2015690575), by MINECO Grant No. FPA2016-76005-C2-1-P and by Maria de Maetzu program Grant No. MDM-20140367 of ICCUB. A. A. thanks Conselho Nacional de Desenvolvimento Científico (CNPq) for its financial support, Grant No. 307265/2017-0. We are specially indebted to Juan Gonzalez Fraile for providing us with his personal codes and detailed results relevant to the analysis in Ref. [8].
[1] G. Aad et al. (ATLAS Collaboration), Phys. Lett. B 716, 1 (2012).

[2] S. Chatrchyan et al. (CMS Collaboration), Phys. Lett. B 716, 30 (2012).

[3] The LEP Collaborations (ALEPH, DELPHI, L3, OPAL, and the LEP TGC Working Group), A combination of preliminary results on gauge boson couplings measured by the LEP experiments, http://lepewwg.web.cern.ch/LEPEWWG/ lepww/tgc, LEPEWWG/TGC/2002-02.

[4] G. Aad et al. (ATLAS Collaboration), J. High Energy Phys. 09 (2016) 029.

[5] V. Khachatryan et al. (CMS Collaboration), Eur. Phys. J. C 76, 401 (2016).

[6] G. Aad et al. (ATLAS Collaboration), Phys. Rev. D 93, 092004 (2016).

[7] V. Khachatryan et al. (CMS Collaboration), Eur. Phys. J. C 77, 236 (2017).

[8] A. Butter, O. J. P. Éboli, J. Gonzalez-Fraile, M. C. GonzalezGarcia, T. Plehn, and M. Rauch, J. High Energy Phys. 07 (2016) 152.

[9] F. de Campos, M. C. Gonzalez-Garcia, and S. F. Novaes, Phys. Rev. Lett. 79, 5210 (1997).

[10] T. Corbett, O. J. P. Éboli, J. González-Fraile, and M. C. González-Garcia, Phys. Rev. Lett. 111, 011801 (2013).

[11] T. Corbett, O. J. P. Éboli, D. Goncalves, J. GonzálezFraile, T. Plehn, and M. Rauch, J. High Energy Phys. 08 (2015) 156.

[12] T. Corbett, O. J. P. Éboli, J. González-Fraile, and M. C. González-Garcia, Phys. Rev. D 86, 075013 (2012).

[13] A. Falkowski, M. Gonzalez-Alonso, A. Greljo, and D. Marzocca, Phys. Rev. Lett. 116, 011801 (2016).

[14] A. Falkowski, M. Gonzalez-Alonso, A. Greljo, D. Marzocca, and M. Son, J. High Energy Phys. 02 (2017) 115.

[15] J. de Blas, M. Ciuchini, E. Franco, S. Mishima, M. Pierini, L. Reina, and L. Silvestrini, Proc. Sci., EPS-HEP2017 (2017) 467 [arXiv:1710.05402].

[16] J. Ellis, C. W. Murphy, V. Sanz, and T. You, J. High Energy Phys. 06 (2018) 146.

[17] Z. Zhang, Phys. Rev. Lett. 118, 011803 (2017).
[18] J. Baglio, S. Dawson, and I. M. Lewis, Phys. Rev. D 96, 073003 (2017).

[19] S. Schael et al. (ALEPH, DELPHI, L3, OPAL, and SLD Collaborations, The LEP Electroweak Working Group, and The SLD Electroweak and Heavy Flavour Groups), Phys. Rep. 427, 257 (2006).

[20] L. E. W. Group (ALEPH, CDF, D0, DELPHI, L3, OPAL, and SLD Collaborations, The LEP Electroweak Working Group, The Tevatron Electroweak Working Group, and The SLD Electroweak and Heavy Flavour Groups), arXiv:1012.2367.

[21] G. Aad et al. (ATLAS and CMS Collaborations), Phys. Rev. Lett. 114, 191803 (2015).

[22] W. Buchmuller and D. Wyler, Nucl. Phys. B268, 621 (1986).

[23] B. Grzadkowski, M. Iskrzynski, M. Misiak, and J. Rosiek, J. High Energy Phys. 10 (2010) 085.

[24] H. D. Politzer, Nucl. Phys. B172, 349 (1980).

[25] H. Georgi, Nucl. Phys. B361, 339 (1991).

[26] C. Arzt, Phys. Lett. B 342, 189 (1995).

[27] H. Simma, Z. Phys. C 61, 67 (1994).

[28] K. Hagiwara, S. Ishihara, R. Szalapski, and D. Zeppenfeld, Phys. Rev. D 48, 2182 (1993).

[29] K. Hagiwara, T. Hatsukano, S. Ishihara, and R. Szalapski, Nucl. Phys. B496, 66 (1997).

[30] T. Corbett, O. J. P. Éboli, J. González-Fraile, and M. C. González-Garcia, Phys. Rev. D 87, 015022 (2013).

[31] A. De Rujula, M. B. Gavela, P. Hernandez, and E. Masso, Nucl. Phys. B384, 3 (1992).

[32] J. Elias-Miro, J. R. Espinosa, E. Masso, and A. Pomarol, J. High Energy Phys. 11 (2013) 066.

[33] T. Corbett, O. J. P. Éboli, and M. C. Gonzalez-Garcia, Phys. Rev. D 96, 035006 (2017).

[34] J. Alwall, R. Frederix, S. Frixione, V. Hirschi, F. Maltoni, O. Mattelaer, H. S. Shao, T. Stelzer, P. Torrielli, and M. Zaro, J. High Energy Phys. 07 (2014) 079.

[35] N. D. Christensen and C. Duhr, Comput. Phys. Commun. 180, 1614 (2009).

[36] A. Alloul, N. D. Christensen, C. Degrande, C. Duhr, and B. Fuks, Comput. Phys. Commun. 185, 2250 (2014). 
[37] T. Sjostrand, S. Mrenna, and P. Z. Skands, J. High Energy Phys. 05 (2006) 026.

[38] J. de Favereau, C. Delaere, P. Demin, A. Giammanco, V. Lemaitre, A. Mertens, and M. Selvaggi (DELPHES 3 Collaboration), J. High Energy Phys. 02 (2014) 057.
[39] C. Patrignani et al. (Particle Data Group), Chin. Phys. C 40, 100001 (2016).

[40] M. Ciuchini, E. Franco, S. Mishima, M. Pierini, L. Reina, and L. Silvestrini, Nucl. Part. Phys. Proc. 273-275, 2219 (2016). 